\title{
Sequential magnetotunneling in a vertical Quantum Dot tuned at the crossing to higher spin states
}

\author{
B. Jouault \\ INFM Unitá di Napoli, Mostra d'Oltremare, Pad 20, 80125 Napoli (Italy) \\ L2M, CNRS,Bagneux,(France) \\ G. Santoro \\ INFM and Scuola Internazionale Superiore di Studi Avanzati (SISSA), Via Beirut 4, 34013 Trieste (Italy) \\ A. Tagliacozzo \\ INFM Unitá di Napoli, Mostra d'Oltremare, Pad 20, 80125 Napoli (Italy) \\ Institut für Nanotechnologie (INT), Forschungszentrum, Postfach 3640, D-76021 Karlsruhe (Germany)
}

\begin{abstract}
We have calculated the linear magnetoconductance across a vertical parabolic Quantum Dot with a magnetic field in the direction of the current. Gate voltage and magnetic field are tuned at the degeneracy point between the occupancies $N=2$ and $N=3$, close to the Singlet-Triplet transition for $N=2$. We find that the conductance is enhanced prior to the transition by nearby crossings of the levels of the 3 particle dot. Immediately after it is depressed by roughly $1 / 3$, as long as the total spin $S$ of the 3 electron ground state doesn't change from $S=1 / 2$ to $S=3 / 2$, due to spin selection rule. At low temperature this dip is very sharp, but the peak is recovered by increasing the temperature.
\end{abstract}

PACS number(s): 71.30.+h, 72.15.Gd, 75.30.Kz, 72.15.Qm

\section{INTRODUCTION}

The conductance of a clean vertical Quantum Dot (QD) versus a gate voltage $V_{g}$ and a source-drain voltage $V_{s d}$ allows the detailed study of the ground state (GS) and first excited states of few electrons confined in the dottit (see fig. 1). The discrete levels due to the parabolic confining potential introduce a shell structure in the electron filling. In the case of a circular disk, application of a magnetic field $B$ in the direction of the current (z-direction), orthogonal to the dot, favours states with increasing angular momenta, as well as higher total spin, also in the absence of Zeeman spin splitting which is believed to be small in these systems the crossings depends on the ratio between the Coulomb energy scale $U$ and the level spacing of the confinement potential $\hbar \omega_{d}$. We consider here the case $U \sim \hbar \omega_{d}$. For larger ratios, transitions with $B$ to higher spin states in dots with few electrons have been related to an "electron molecule" picture for the particle distribution within the dot 6 .

These features are strongly dependent on the long ranged interaction. Therefore, exactly soluble models like the Constant Interaction (CI) model or even the one with harmonic e-e interaction (HI) are unable to reproduce them . In fact, the HI model allows only for ground states which are singlet (S) for $N$ even ( $N$ is the number of electrons in the isolated dot) or doublet (D) for $N$ odd. On the contrary, it was long ago recognized that a two-electron dot undergoes a Singlet-Triplet (S-T) transition when the magnetic field is large enough $\mathrm{l}$. This was indeed seen2 at $B_{S T} \sim 4$ Tesla in dots whose confining energy $\hbar \omega_{d}$ is about $5 \mathrm{meV}$. In fact, at difference with what happens in the ${ }^{4} \mathrm{He}$ atom $\left(B_{S T} \sim 10^{5} \mathrm{Tesla}\right)$, here the $\mathrm{S}-\mathrm{T}$ energy separation at $B=0$ is only $\sim 5 \mathrm{meV}$. DoubletQuadruplet (D-Q) transitians for an $N=3$ particle dot are correspondingly found (see below). Effects on the conductance are expected which are usually named "spin blockade" phenomena10:

a) if the total spin of the ground state of the $N+1$ and $N$ particle $\operatorname{dot}\left({ }^{N+1} G S,{ }^{N} G S\right.$ in the following) differ by more than $1 / 2$, the dot is blocked, with the corresponding peak in the linear conductance missing at zero temperature.

b) The reduction of the total spin in adding an extra electron to the dot has a lower transition rate as compared to spin increase. This can lead to negative differential conductance.

In this work we analyze in detail the behavior of the linear conductance of the dot when the gate voltage is such that the GS energies for $N=2$ and $N=3$ are degenerate (see fig. 1] $\mathbf{b}$ ) and we show that, by increasing $B$ beyond the S-T transition of the $N=2$ particle dot, the conductance displays a sudden dip due to the spin selection rule $b$ ). Because the corresponding ${ }^{3} G S$ has total spin $1 / 2$, the conductance is depressed by roughly $1 / 3$. In fact, due to conservation of $S_{z}$, it is impossible to add an electron with spin up (down) to the $N=2$ particle dot in the triplet state with $S_{z}=1(-1)$, to give total spin $S=1 / 2$. These two (over six) possibilities that are missing are restored when the ${ }^{3} G S$ changes to $S=3 / 2$. At low temperatures this dip is very sharp, but the peak is recovered in increasing the temperature.

Three main ingredients are required to calculate the linear conductance, according to second order perturbation theory in the tunneling:

i) the energies ${ }^{N} E_{\alpha}$ of the isolated dot with e-e interactions (where $\mathrm{N}=2,3$ and $\alpha$ labels the states of the dot). 
(a)

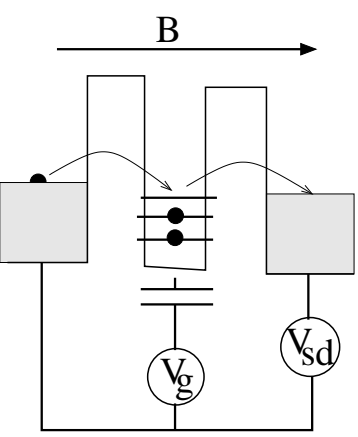

studied point

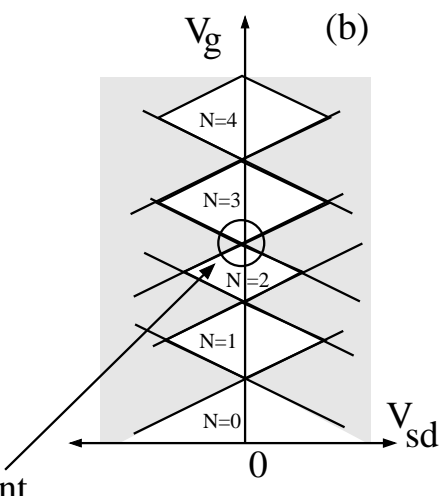

$\mathrm{V}_{\mathrm{sd}}$

FIG. 1. (a)Sketch of the conduction band of the device in a linear transport experiment with a magnetic field B along the direction of the current. (b) Grey-scale conductance plot in the plane $\left(V_{g}, V_{s d}\right)$. The circle encloses the conductance peak at the degeneracy point $E_{0}^{(N=3)}\left(V_{g}, B\right)=E_{0}^{(N=2)}\left(V_{g}, B\right)$ studied in this work.

ii) the spectral amplitude $Z^{N+1, \alpha}$ for electron addition to the dot.

iii) the one-particle tunneling matrix $\Gamma$ from the contacts to the dot.

The first two quantities are provided by exact diagonalization of the isolated dot, which uses a Lanczos code (up to 7 electrons), already employed previously in the analysis of the magnetoconductance data in pillar strug tures 11 . The third quantity was calculated analytically 12 . In Section II we study the transition rates in a dot from $N=2$ to $N=3$ by addition of an extra electron in an orbital of given quantum numbers. We analyze the various contributions to the tunneling, and the details of the spectral amplitude around $B_{S T}$. We find that the spin selection rule mentioned above is responsible for a marked difference in the peak heights for transitions from ${ }^{2} T^{1} \rightarrow{ }^{3} D^{1,2}$ and ${ }^{2} T^{1} \rightarrow{ }^{3} Q^{3}$ (see table I for the notation). The linear magnetoconductance displays a sharp dip at low temperature at the S-T transition. In Section III we comment the results: similar peculiarities of the energy spectrum around $B_{S T}$ can be found for larger $N(N>3)$. They are remnant of the S-T transition in the $N=2$ particle dot. However, in increasing $N$, the number of crossings is expected to increase and they occur in a smaller energy range, so that their effect on the transition rate could be averaged out by temperature.

\section{LINEAR MAGNETOCONDUCTANCE AT THE S-T TRANSITION}

Transitions of an isolated dot to higher spin states with increasing magnetic field, follow from an interplay between the e-e interaction and the confining potential. They are easily monitored with exact diagonalization of the electron system. In fig. 2 the position in energy of the first levels of a two-dimensional(2D) QD for $N=2$

and $N=3$ is plotted versus magnetic field, expressed in $\hbar \omega_{c}$. Here $\omega_{c}=e B / m^{*} c$ is the cyclotron frequency and $m^{*}=0.067 m_{e}$ is the effective electron mass in GaAs. States are labeled by the total spin $S$ and by the components (along the direction of the field $B$ ) of the total spin $S_{z}$ and of the total angular momentum $M$ (see Table I).

The dot is laterally confined by a parabolic potential with level spacing $\hbar \omega_{d}=4 \mathrm{meV}$. The e-e interaction is $U \times l_{d} /\left|\mathbf{r}-\mathbf{r}^{\prime}\right|$, where $l_{d}=\sqrt{\hbar / m^{*} \omega_{d}}$ is the length scale and the vectors are in the dot plane. The energy scale of the Coulomb interaction $U$ is taken equal to $3 \mathrm{meV}$. Spin splitting is not included in the picture for clarity. It is $g^{*} \mu_{B} B \sim 0.1 \mathrm{meV}$ ( $\mu_{B}$ is the Bohr magneton) at these fields if the bulk gyromagnetic ratio for $G a A s\left(g^{*}=-0.44\right)$ is assumed, but it could be lower 13. A Lanczos algorithm is used on a basis which includes Slater determinants constructed with up to 28 single particle orbitals that are solutions of the $2 \mathrm{D}$ harmonic confining potential.

The S-T crossing in the ${ }^{2} G S$ (fig. $2 \mathbf{a}, \mathbf{c}$ ) occurs at $\hbar \omega_{c} \sim$ $7.5 \mathrm{meV}$. The next available levels are also shown and are located rather higher in energy. Correspondingly, the ${ }^{3} G S$ (fig. $2 \mathbf{b}, \mathbf{d}$ ) is the doublet ${ }^{3} D^{1}$ at lower $\hbar \omega_{c}$ and becomes the doublet ${ }^{3} D^{2}$ at $\hbar \omega_{c}=7.8 \mathrm{meV}$, according to the general rule that higher magnetic fields favor larger angular momenta. Next, there is a doubletquadruplet (D-Q) transition at the magnetic field $B_{D Q}$

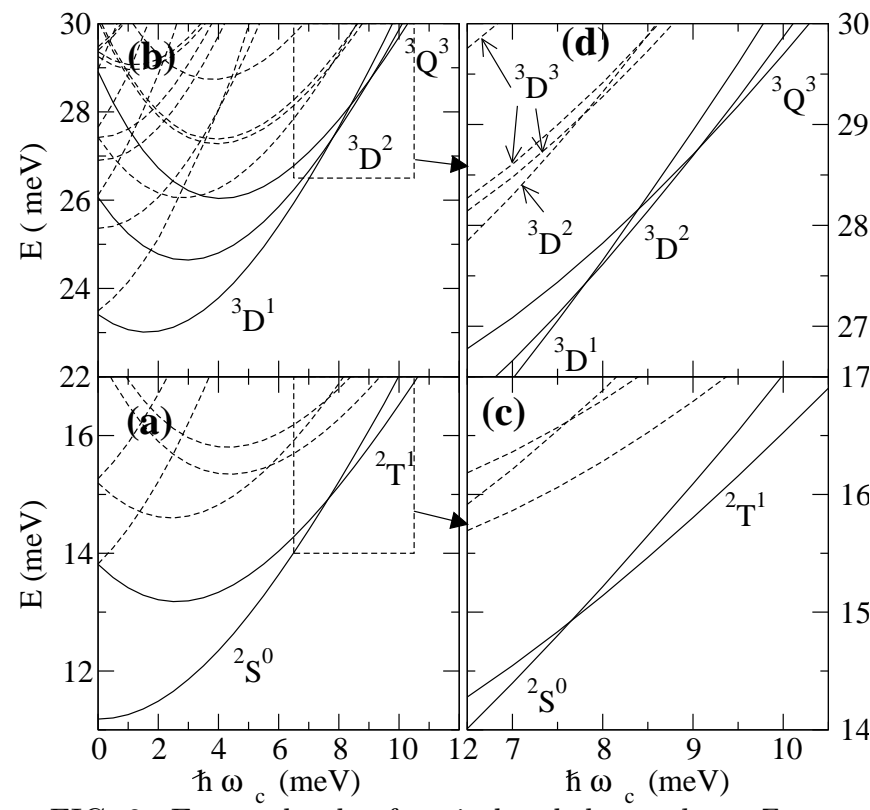

FIG. 2. Energy levels of an isolated dot vs $\hbar \omega_{c}$. Zeeman spin splitting is not included. (a) and (c): dot with 2 electrons. (b) and (d): dot with 3 electrons. Fig.s (c) and (d) magnify the rectangle areas of $(\mathbf{a})$ and $(\mathbf{b})$ respectively. The level spacing of the confining potential is $\hbar \omega_{d}=4 \mathrm{meV}$, the strength of the Coulomb interaction is $U=3 \mathrm{meV}$. The GS levels ${ }^{2} S,{ }^{2} T,{ }^{3} D^{1},{ }^{3} D^{2},{ }^{3} Q^{3}$ are represented by solid lines (notation in Table I). Higher excited levels are represented by dashed lines. 


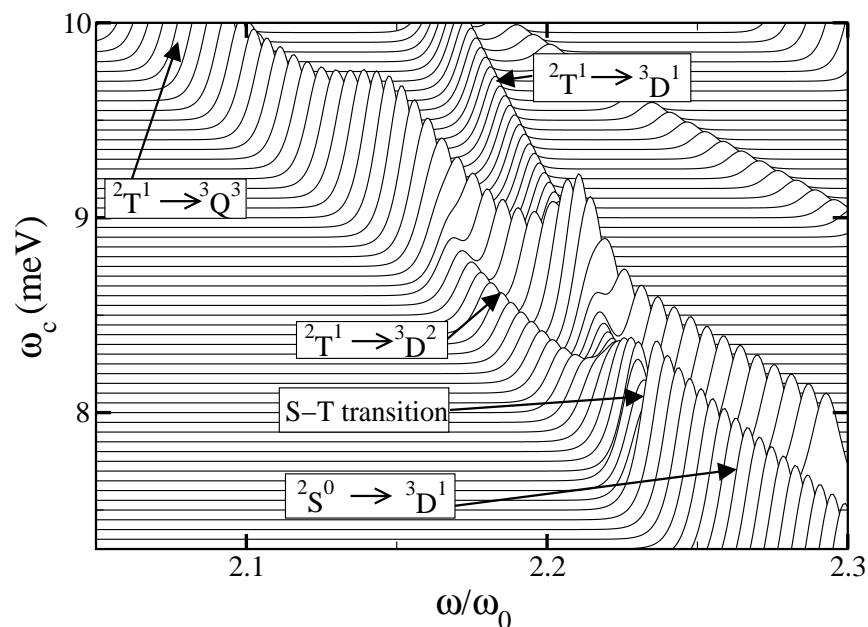

FIG. 3. Transition rates as a function of $\omega / \omega_{0}$ where $\hbar \omega=E-{ }^{2} E_{0}$ is the energy difference with respect to the ${ }^{2} G S$ energy $\left(\omega_{0}=\sqrt{\omega_{d}^{2}+\omega_{c}^{2} / 4}\right)$. Zeeman spin splitting is not included. The step in $\hbar \omega_{c}$ is equal to $0.05 \mathrm{meV}$. Curves are shifted for clarity. A thermal broadening of $T \sim 200 \mathrm{mK}$ has been added. The dot parameters are $\hbar \omega_{d}=4 \mathrm{meV}, \mathrm{U}=3 \mathrm{meV}$.

$\left(\hbar \omega_{c} \sim 9 \mathrm{meV}\right)$. Therefore, the sequence of transitions with increasing $B$, for addition of one electron at the lowest energy cost, is the following: ${ }^{2} S \rightarrow{ }^{3} D^{1},{ }^{2} T \rightarrow{ }^{3} D^{1}$, ${ }^{2} T \rightarrow{ }^{3} D^{2},{ }^{2} T \rightarrow{ }^{3} Q^{3}$. The ${ }^{3} D^{1} \rightarrow{ }^{3} D^{2}$ transition always occurs at a higher magnetic field than the S-T transition in the ${ }^{2} G S$ : this has been numerically verified by varying the confinement of the dot.

We calculate the transition rates for addition of one extra electron $(n m \sigma)$ from the contacts. $n$ and $m$ are the principal quantum number and the angular momentum which label the one-particle energies $\epsilon_{n m}=\hbar \omega_{0}(n+$ $1)+m \hbar \omega_{c} / 2\left(\omega_{0}=\sqrt{\omega_{d}^{2}+\omega_{c}^{2} / 4}\right)$. Here $m$ has the same parity as $n$, taking the values $-n,-n+2, \ldots, n-2, n$.

The transition rate reads:

$$
\begin{array}{r}
t_{N, 0}^{N+1, \alpha}(\epsilon)=\sum_{n m, n^{\prime} m^{\prime}} \Gamma_{n m}^{n^{\prime} m^{\prime}}(\epsilon) Z_{n m \sigma}^{N+1, \alpha}\left[Z_{n^{\prime} m^{\prime} \sigma}^{N+1, \alpha}\right]^{*} \\
\delta\left(\epsilon-\left(E_{\alpha}^{N+1}-E_{0}^{N}\right)\right) .
\end{array}
$$

Here $Z_{n m \sigma}^{N+1, \alpha}=\left\langle N+1, \alpha\left|c_{n m \sigma}^{\dagger}\right| N, 0\right\rangle$ is the spectral weight amplitude. The dot is assumed to be in the ${ }^{N} G S$, $|N 0\rangle$, prior to electron addition and $\alpha \equiv(S, M)$ labels the final state with $N+1$ electrons.

The matrix $\Gamma_{n m}^{n^{\prime} m^{\prime}}$ appearing in eq. 1 1 contains the single particle orbital overlaps. It describes the coupling of the dot to the contacts which have highly degenerate Landau subbands and is factorized in the $z$ and orthogonal directions. It is also diagonal in the $m$ indices, as the component of the angular momentum in the $z$ - direction is conserved in the tunneling due to the assumed cylindrical symmetry. Peaks in the transition rate of eq. 1 are present each time the energy $\epsilon$ matches $\mu_{N, 0}^{N+1, \alpha} \equiv{ }^{N+1} E_{\alpha}-{ }^{N} E_{0}$, provided conservation of $S, S_{z}$ and $M$ are assured when adding the extra electron. We neglect Zeeman spin splitting: its effect will be discussed shortly in the conclusion. The transition rates are plotted in fig. 3 vs the dimensionless ratio $\omega / \omega_{0}$ for increasing magnetic field. Here $\hbar \omega=E-{ }^{2} E_{0}$ is the excitation energy with respect to the ${ }^{2} G S$. Curves (at intervals of $\Delta \hbar \omega_{c}=0.05 \mathrm{meV}$ ) are shifted upwards for clarity with increasing $\omega_{c}$. An extra width has been given to the peaks (which are $\delta$-like in eq. (1), what is consistent with a thermal broadening of $200 \mathrm{mK}$ (see below). Each series of peaks corresponds to the addition of one particle of definite angular momentum to the ${ }^{2} G S$. As an example, the peak from the singlet GS at $\omega / \omega_{0} \approx 2.3$, for the lowest magnetic field, corresponds to the addition of one electron with $m=1$, what changes the state of the dot from ${ }^{2} S^{0}$ to ${ }^{3} D^{1}$. The initial and final states of the transition are labeled within the figure. The landscape changes drastically when the ${ }^{2} G S$ becomes a triplet.

In the plot of fig. 3 only the diagonal term $n=n^{\prime}$ has been included in the sums of eq. 1. This is expected to give the largest contribution within weak coupling 14 . The spectral weight $\left|Z_{n m \sigma}^{N+1, \alpha}\right|^{2}$ is largely responsible for the heights of the peaks in fig. 3 .

This is seen in fig. $1 \mathbf{a}, \mathbf{a}, \mathbf{b}$, for the case when an electron $n=2, m=2$ is added to the ${ }^{2} T_{1}^{1}$ state (denoted symbolically as $\left.C_{22 \sigma}^{\dagger}\right|^{2} T_{1}^{1}>$ ), producing the final state with $M=3$. There are four low lying states in the $\mathrm{N}=3$ energy spectrum, labeled ${ }^{3} Q^{3}$ and ${ }^{3} D^{3}$ (fig. $4 \mathbf{a}$ ). The lowest is the quadruplet state ${ }^{3} Q^{3}$. The spectral weight for addition of an electron with spin down is distributed among them (r.h.s. of fig. $1 \mathbf{a}$ and fig. $4 \mathbf{c}$ ), while

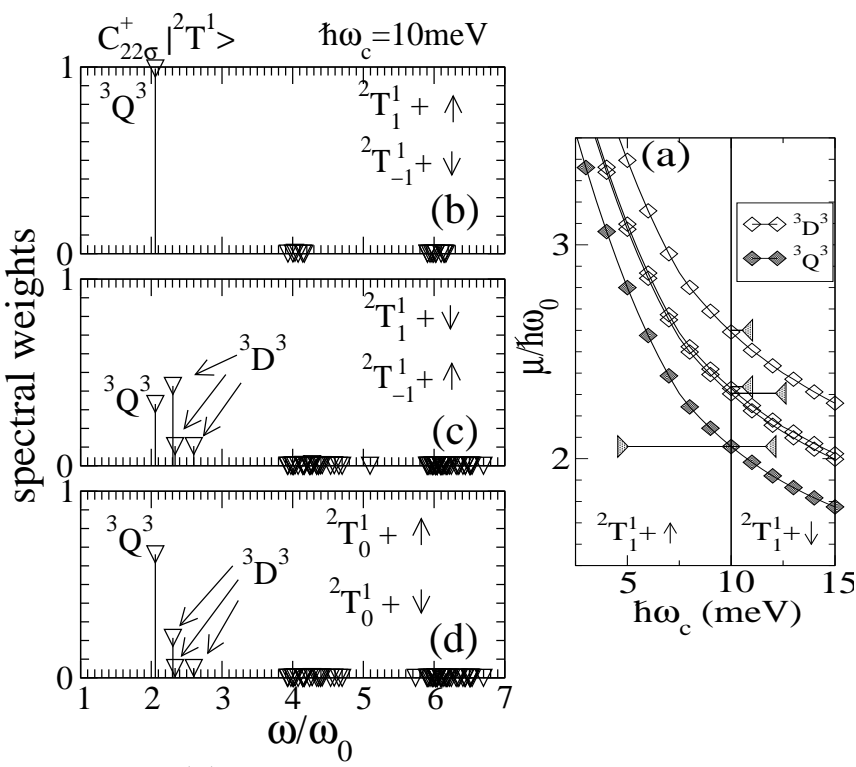

FIG. 4. (a): The energy levels with $M=3$ vs $\hbar \omega_{c}$ are plotted on the right side. The spectral weights are depicted as horizontal lines to the right $(+\downarrow)$ and to the $\operatorname{left}(+\uparrow)$ of the vertical line. (b), (c),(d): Spectral weights for the ${ }^{2} T^{1} \rightarrow{ }^{3} Q^{3}$ transition at $\hbar \omega_{c}=10.0 \mathrm{meV}$. $\hbar \omega=\left(E-{ }^{2} E_{0}\right)$ are the excitation energies. Dot parameters as in fig. 3 . 
in the case of a spin up particle the spectral weight is practically exhausted by the transition to the quadruplet state with $S_{z}=3 / 2$ (l.h.s. of fig. Ha and fig. $4 \mathbf{b}$ ). Up and down arrows are to be interchanged in the case of ${ }^{2} T_{-1}^{1}$ 15. Transition rates are proportional to the square of the Clebsch-Gordan coefficients $\left|\left\langle S^{\prime} S_{z}^{\prime} \mid S S_{z} \frac{1}{2} \pm \frac{1}{2}\right\rangle_{C G}\right|^{2}$ which accounts for the spin selection rules 10 .

These results are fully obvious in a picture in which Coulomb interaction is treated as a perturbation.

Starting by filling single particle levels of the parabolic confinement potential, the $N=3$ state (lowest in energy) with $S_{z}=1 / 2$ and $M=3$ is obtained from the linear combination of three degenerate states with two electrons up and one down, distributed between $n=0, m=0 ; n=$ $1, m=1 ; n=2, m=2$. Coulomb interaction breaks the degeneracy, giving rise to the quadruplet state $\left|{ }^{3} Q_{1 / 2}^{3}\right\rangle$, which is the properly antisymmetrized combination of the previous three (with normalizing factor $1 / \sqrt{3}$ ) and two doublet states ${ }^{3} D_{1 / 2}^{3}$, which are almost unsplitted (see fig. $4 \mathbf{a}$ ). The third doublet state at higher energy in fig. Ta is obtained by placing one electron in $n=3, m=3$ and the two others in $n=0, m=0$. The transition ${ }^{2} T_{1}^{1}$ $\rightarrow{ }^{3} Q_{1 / 2}^{3}$ by addition of one electron of spin $\downarrow$ has the weight $\left|Z_{22 \downarrow}\right|^{2} \sim 1 / 3$ :

$$
\left.\left|<{ }^{3} Q_{1 / 2}^{3}\right| C_{22 \downarrow}^{\dagger}\right|^{2} T_{1}^{1}>\left.\right|^{2}=\mid\left\langle{ }^{3} Q_{1 / 2}^{3}\left|C_{22 \downarrow}^{\dagger} C_{11 \uparrow}^{\dagger} C_{00 \uparrow}^{\dagger}\right| 0>\left.\right|^{2}=\frac{1}{3} .\right.
$$

This spectral weight corresponds to the first peak of fig. [4c. Similarly, the transition ${ }^{2} T_{0}^{1} \rightarrow{ }^{3} Q_{1 / 2}^{3}$ has a weight $\left.\left|Z_{22 \uparrow}\right|^{2} \approx\left|<{ }^{3} Q_{1 / 2}^{3}\right| C_{22 \uparrow}^{\dagger}\right|^{2} T_{0}^{1}>\left.\right|^{2}=2 / 3$ (fig. 田 $\mathbf{d}$ ). Finally the transition ${ }^{2} T_{1}^{1} \rightarrow{ }^{3} Q_{3 / 2}^{3}$ has a spectral amplitude $\left.\left|Z_{22 \uparrow}\right|^{2} \approx\left|<{ }^{3} Q_{3 / 2}^{3}\right| C_{22 \uparrow}^{\dagger}\right|^{2} T_{1}^{1}>\left.\right|^{2}=1$ (fig. 那). Comparing these noninteracting estimates with the exact results, the agreement is found to be close.

To study the linear magnetoconductance we use a very general expression for the current derived in the tunneling Hamiltonian formalism 16 . If the coupling between the left $(\mathrm{L})$ and right $(\mathrm{R})$ barrier is proportionate, the linear conductance can be written in terms of the transition rates of eq. 1:

$$
g_{N, 0}\left(e V_{g}\right)=\left.\frac{d I}{d V}\right|_{N, 0}\left(e V_{g}\right) \propto \frac{e^{2}}{\hbar} \sum_{\alpha} \int_{0}^{\infty} d \epsilon t_{N, 0}^{N+1, \alpha}(\epsilon) \frac{\partial f}{\partial \epsilon},
$$

where $f$ is the Fermi function. Just the diagonal terms are retained in eq. I (what is found to provide the largest contribution by weak coupling, as above mentioned). Peaks are present when the gate voltage with respect to $\mu$ is such that $e V_{g}=\mu_{N, 0}^{N+\alpha}$. In the absence of crossings, only the GS $(\alpha=0)$ contributes to the linear conductance. Indeed, other $\alpha$ 's would correspond to a nonequilibrium condition for the dot. Close to the S-T crossing, the conduction will be the sum of the two contributions $g_{S}$ and $g_{T}$, weighted by the canonical equilibrium prob-

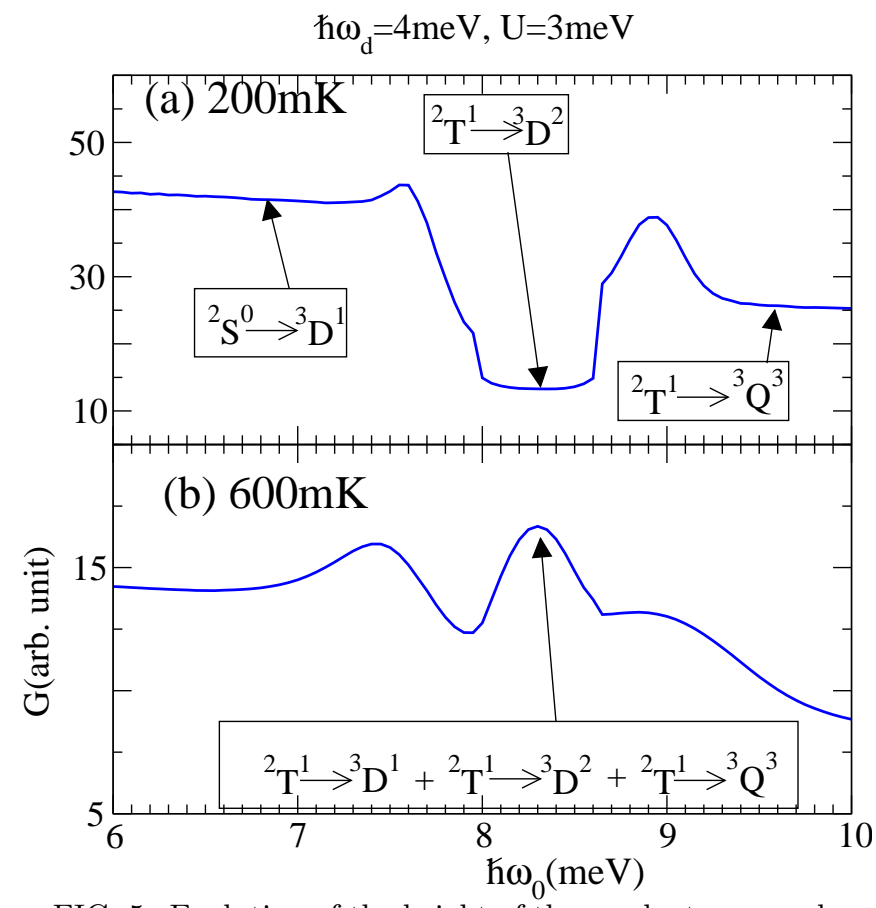

FIG. 5. Evolution of the height of the conductance peak as a function of $\hbar \omega_{c}$. (a) At a temperature of $200 \mathrm{mK}$, a dip is clearly visible in the range $\hbar \omega_{c}=8-8.8 \mathrm{meV}$. (b) When the temperature increases, the dip leaves place to a peak which is due to the contributions of the ${ }^{2} T^{1} \rightarrow{ }^{3} D^{1}$ and ${ }^{2} T^{1} \rightarrow{ }^{3} Q^{3}$ transitions.

ability of the dot being in the singlet or the triplet ${ }^{2} \mathrm{GS}$ $\left(P_{S}\right.$ and $P_{T}^{\zeta}$ respectively, with $\left.\zeta \equiv S_{z}= \pm 1,0\right)$ 1]:

$$
\left.\frac{d I}{d V}\right|_{V_{s d}=0}\left(e V_{g}\right)=P_{S} \cdot g_{S}+\sum_{\zeta= \pm 1,0} P_{T}^{\zeta} \cdot g_{T}^{\zeta}
$$

In fig. 5, the maximum of the linear magnetoconductance calculated using eq. 2 is shown at increasing magnetic fields for two values of the temperature (fig. 3a: $T=200 \mathrm{mK}$, fig. $3 \mathbf{b}: T=600 \mathrm{mK})$. At the lowest temperature it displays sharp features close to $B_{S T}$ and a marked dip between $B_{S T}$ and $B_{D Q}$. Conductance is at a maximum when the ${ }^{2} \mathrm{GS}$ is a singlet while the ${ }^{3} \mathrm{GS}$ is a doublet. The same happens at $B>B_{D Q}$ when the ${ }^{2} \mathrm{GS}$ is a triplet while the ${ }^{3} \mathrm{GS}$ is a quadruplet. In the latter case, there are six tunneling channels corresponding to ${ }^{2} \mathrm{GS}$ having $S_{z}= \pm 1,0$ and addition of one extra electron with up or down spin. Each of them contributes $1 / 6$ to the total conductance. Between $B_{S T}$ and $B_{D Q}$, however, the ${ }^{3} \mathrm{GS}$ is still a doublet, what inhibits two of the possible tunneling channels.

Increasing temperature (fig. $5 \mathbf{b}$ ), there is some overlap between the peaks due to the two doublet states ${ }^{3} D^{1}$ and ${ }^{3} D^{2}$ and the state ${ }^{3} Q^{3}$ as well, which produces larger conduction in the intermediate region between $B_{S T}$ and $B_{D Q}$ and a new peak appears. This eventually washes out the dip. 


\section{CLOSING REMARKS}

The peak height in Coulomb blockade oscillations versus gate voltage $V_{g}$ is governed by the ${ }^{N} G S \rightarrow{ }^{N+1} G S$ transition rate. Using the $\mathrm{S}-\mathrm{T}$ transition for the $N=2$ particle dot as an example, we have shown that, in the neighborhood of $B_{S T}$, crossing of levels are to be expected for the $N+1$ particle dot as well. This feature seems to be general, as seen from the dot spectrum with $N=4$ and $N=5$. Increasing the ratio of the Coulomb energy $U$ to the level spacing $\hbar \omega_{d}$ due to the confinement potential, moves these crossings to lower magnetic fields.

We have calculated the transition rates for addition of one electron to the dot with $N=2$ in an orbital with quantum numbers $n m \sigma$. They are non-zero at the discrete energies $\epsilon=\mu_{N, 0}^{N+1, \alpha}$. While the single particle overlap $\Gamma(\epsilon)$ plays a minor role, transition rates are determined in weak coupling by the spectral amplitude for the isolated dot, which we obtain by exact diagonalization of a 2D system with parabolic confinement and Coulomb interaction between the electrons.

Previous numerical work on the spectral weight of a few electron dot was mostly concerned with the total quantity $w_{\alpha}=\sum_{n m \sigma}\left|Z_{n m \sigma}^{N+1, \alpha}\right|^{2}$ (seeg for $B=0$ and 18 for $\alpha=0$ at various $B$ ), in which the spectral weights are summed over all possible orbitals for the added electron. They showed that at definite energies its value could be strongly reduced with respect to the noninteracting case, due to correlations. However, in a vertical configuration for tunneling, with $B$ in the direction of the current, transition rates for the dot from the ${ }^{N}$ GS to the ${ }^{N+1} \mathrm{GS}$, or closely lying excited states at $N+1$, require conservation of the total angular momentum $M$ in the direction of the field, as well as of the total spin component $S_{z}$.

The quantity $w_{\alpha}$ is then of little use, if conservation of the quantum numbers mentioned before has to be enforced. We have shown that each of the contribution to $w_{\alpha}$, i.e. each of the spectral amplitudes, is strongly dependent on the quantum numbers which label the dot states and on the single particle orbital of the electron tunneling onto the dot.

The spin selection rule we have been discussing in Section II arises from conservation of $S_{z}$ in a vertical geometry. The absence of the quasiparticle (QP) peak for addition of an electron of a given spin shows that there are some tunneling processes that cannot contribute to the conduction. This produces a dip in the linear magnetoconductance, in the range of magnetic field values for which the ${ }^{2} G S \rightarrow{ }^{3} G S$ transition is between a triplet $(S=1)$ and a doublet state $(S=1 / 2)$.

As long as the Zeeman spin splitting is ignored, this mechanism is not spin sensitive, because $T_{\zeta}$ with $\zeta=$ $0, \pm 1$ are degenerate in energy. However, if Zeeman spin splitting becomes relevant, the ${ }^{3} Q_{3 / 2}^{3}$ state could become lower in energy than the ${ }^{2} D_{1 / 2}^{2}$ and the range of magnetic field values between $B_{S T}$ and $B_{D Q}$, in which the dip in the conductance occurs, could disappear.
Increased correlation, by distributing weight out of the QP peak to nearby energies, could reduce the effect. This is not the case, however, because we find that much larger $U$ values do not change fig. A qualitatively. Instead, they shift the magnetic field at which the crossings take place, and, of course, increase the energy differences between the levels. We do not find a sizeable spreading of the spectral weight to higher energies for $U$ values as large as $U=15 \mathrm{meV}$. This could be partly due to the limited number of orbitals used in the single particle basis, however.

We acknowledge useful discussions with A. Angelucci, M.Di Stasio, G. Faini, R. Haug, J. Weis. One of us (A.T.) is grateful to G. Schön for the hospitality at the Institut für Festkörperphysik, Universität Karlsruhe, where this work was written. Work partially supported by INFM (Pra97-QTMD) and by EEC with TMR project, contract FMRX-CT98-0180.

${ }^{1}$ S. Tarucha, D. G. Austing, T. Honda, R. J. van der Hage and L. P. Kouwenhoven, Phys. Rev. Lett. 77, 3613 (1996).

${ }^{2}$ L. P. Kouwenhoven, T. H. Oosterkamp, M. W. S. Danoesastro, M. Eto, D. G. Austing, T. Honda and S. Tarucha, Science 278, 1788 (1997).

${ }^{3}$ S. M. Cronenwett, T. H. Oosterkamp and L. P. Kouwenhoven, Science 281, 540 (1998).

${ }^{4}$ J. Weis, R. J. Haug, K. v. Klitzing and K. Ploog, Phys. Rev. Lett. 71, 4019 (1993).

${ }^{5}$ P. A. Maksym and T. Chakraborty, Phys. Rev. Lett. 65, 108 (1990). P. A. Maksym and T. Chakraborty, Phys. Rev. B 45, 1947 (1992). S. R. E. Yang, A. H. MacDonald and M. D. Johnson, Phys. Rev. Lett. 71, 3194 (1993).

${ }^{6}$ H. Imamura, H. Aoki and P. A. Maksym, Phys. Rev. B 57, R4257 (1998). For transitions at $B=0$, in the low density limit, see R. Egger, W. Häusler, C. H. Mak and H. Grabert, Phys. Rev. Lett. 82, 3320 (1999) and Phys. Rev. Lett. 83, 462(E) (1999).

${ }^{7}$ N. S. Johnson and M. C. Payne, Phys. Rev. B 45, 8819 (1992). A. Angelucci and A. Tagliacozzo, Phys. Rev. B 56, R7088 (1997).

${ }^{8}$ M. Wagner, U. Merkt, A. V. Chaplik, Phys. Rev. B 45, 1951 (1992). P. Hawrylak, Phys. Rev. Lett. 71, 3347 (1993).

${ }^{9}$ D. Pfannkuche and S. E. Ulloa, Phys. Rev. Lett. 74, 1194 (1995).

${ }^{10}$ D. Weinmann, W. Häusler, W. Pfaff, B. Kramer and U. Weiss, Europhys. Lett. 26, 467(1994). D. Weinmann, W. Häusler and B. Kramer, Phys. Rev. Lett. 74, 984 (1995). D. Weinmann, W. Häusler and B.Kramer, Annalen der Physik 5, 652 (1996).

${ }^{11}$ B. Jouault, G. Faini, A. Angelucci, M. Di Stasio, G. Santoro, A. Tagliacozzo, F. Laruelle, R. Werner and A. Forchel, cond-mat/9810094.

12 B. Jouault, M. Boero, J. C. Inkson, Phys. Rev. B 45, 1951 (1999). 
${ }^{13}$ M. Dobers, K. v. Klitzing and G. Weinmann, Phys.Rev B38, 5453 (1988).

${ }^{14}$ Quing-feng Sun and Tsung-han Lin, Physica E4, 201 (1999).

${ }^{15}$ The contribution of the orbital with $n=m$ is the most important one. Indeed, the spectral weight corresponding to $n>m$ is usually very dispersed among many energies, except when the quasiparticle (QP) peak survives intact 9 , but the excitation of the center of mass variable takes simultaneously place. This implies that it is located at higher energies anyhow.

${ }^{16}$ Y. Meir and N. S. Wingreen, Phys. Rev. Lett. 68, 2512 (1992). A. Jauho, N. S. Wingreen and Y. Meir, Phys. Rev. B 50, 5528 (1994).

${ }^{17}$ C. W. J. Beenakker, Phys. Rev. B 44, 1646 (1991)

18 J. J. Palacios, L. Martin Moreno, G. Chiappe, E. Louis, C. Tejedor, Phys. Rev. B 50, 5760 (1994).

\begin{tabular}{ll}
\hline \hline \multicolumn{1}{c}{ level } & notation \\
\hline $\mathrm{N}=2, \quad \mathrm{~S}=0, \mathrm{M}=0 \quad$ (singlet) & ${ }^{2} S^{0}$ \\
$\mathrm{~N}=2, \quad \mathrm{~S}=1, S_{z}, \mathrm{M}=1$ (triplet) & ${ }^{2} T_{S_{z}}^{1}$ \\
$\mathrm{~N}=3, \quad \mathrm{~S}=1 / 2, S_{z}, \mathrm{M}$ (doublet) & ${ }^{3} D_{S_{z}}^{M}$ \\
$\mathrm{~N}=3, \quad \mathrm{~S}=3 / 2, S_{z}, \mathrm{M}=3 \quad$ (quadruplet) & ${ }^{3} Q_{S_{z}}^{3}$ \\
\hline \hline
\end{tabular}

TABLE I. Notations for the discrete levels of the QD used in the text. 